\title{
@creative
}

ISSN 1855-3966 (printed edn.), ISSN 1855-3974 (electronic edn.)

ARS MATHEMATICA CONTEMPORANEA 22 (2022) \#P2.03

https://doi.org/10.26493/1855-3974.2501.4c4

(Also available at http://amc-journal.eu)

\section{LDPC codes from cubic semisymmetric graphs*}

\author{
Dean Crnković (D), Sanja Rukavina (D), Marina Šimac † (D) \\ Department of Mathematics, University of Rijeka, \\ Radmile Matejčić 2, 51000 Rijeka, Croatia
}

Received 10 December 2020, accepted 16 July 2021, published online 14 April 2022

\begin{abstract}
In this paper we study LDPC codes having cubic semisymmetric graphs as their Tanner graphs. We discuss the structure of the smallest absorbing sets of these LDPC codes. Further, we give the expression for the variance of the syndrome weight of the constructed codes, and present computational and simulation results.
\end{abstract}

Keywords: LDPC code, cubic graph, semisymmetric graph.

Math. Subj. Class. (2020): 94B05, 05 C99

\section{Introduction and preliminaries}

Throughout this paper we assume graphs to be finite, simple and connected. For the concepts and notation related to the graph theory and coding theory, we refer the reader to [10] and [15], respectively.

In this paper we use cubic semisymmetric graphs for the construction of LDPC codes. A graph is called a 3-regular graph, i.e. a cubic graph, if every vertex of the graph has the degree equal to three. A graph is edge-transitive (vertex-transitive) if its automorphism group acts transitively on the set of edges (set of vertices). A regular graph is semisymmetric if it is edge-transitive, but not vertex-transitive. It has been proved that every semisymmetric graph is necessarily bipartite with two parts of equal size (see [14]).

Semisymmetric graphs were first studied by Folkman in 1967 (see [12]). He proposed a construction of semisymmetric graphs and constructed the smallest semisymmetric graph with 20 vertices and 40 edges (the Folkman graph). Furthermore, it has been proved that there are no semisymmetric graphs with $2 p$ or $2 p^{2}$ vertices for a prime number $p$.

\footnotetext{
*This work has been fully supported by Croatian Science Foundation under the project 6732 .

${ }^{\dagger}$ Corresponding author.

E-mail addresses: deanc@math.uniri.hr (Dean Crnković), sanjar@math.uniri.hr (Sanja Rukavina), msimac@math.uniri.hr (Marina Šimac)
} 
A cubic semisymmetric graph is a 3-regular graph which is semisymmetric. A construction of cubic semisymmetric graphs and the (non)existence of graphs with a certain number of vertices have been a subject of many studies. For example, in [20], the existence of the unique cubic semisymmetric graph with $2 p^{3}$ vertices for a prime number $p$, the Gray graph of order 54, was proved. In [11], the condition for the existence of cubic semisymmetric graphs with $6 p^{3}$ vertices was given, and a construction of such graphs was described. The classification of cubic semisymmetric graphs with at most 768 vertices was given in [4]. All of the listed graphs have girth at least eight.

The dual code $\mathcal{C}^{\perp}$ of an $[n, k]$ linear code $\mathcal{C}$ is an $[n, n-k]$ code defined by

$$
\mathcal{C}^{\perp}=\left\{x \in \mathbb{F}_{p}^{n} \mid x \cdot y=0, \forall y \in \mathcal{C}\right\}
$$

where is the standard inner product. A generator matrix of the code $\mathcal{C}^{\perp}$ is called a paritycheck matrix of $\mathcal{C}$.

A binary low-density parity-check (LDPC) code is a binary linear code defined by a sparse parity-check matrix $H$. That is to say, $H$ contains a very small number of nonzero entries. An LDPC code is $\left(w_{c}, w_{r}\right)$-regular if the weight of each column is equal to $w_{c}$, and the weight of each row is equal to $w_{r}$.

LDPC codes can be presented using Tanner graphs, which were introduced by Tanner in [26]. The Tanner graph of an LDPC code is a bipartite graph that consists of two sets of vertices; bit nodes that correspond to codeword bits and check nodes that correspond to parity-check equations. An edge connects a bit node to a check node if that bit is included in the corresponding parity-check equation. If an LDPC code is $\left(w_{c}, w_{r}\right)$-regular, the corresponding Tanner graph is a biregular bipartite graph in which vertices are of degree $w_{c}$ or $w_{r}$.

The decoding performance of an LDPC code depends on the structure of the corresponding Tanner graph; the existence of short cycles in the Tanner graph of a code establishes a correlation between iterations in the process of decoding, and therefore, has a negative impact on the bit error rate (BER) performance of the code. The shorter the cycles are, the more significant the effect is. Furthermore, the iterative decoding performance of an LDPC code is related with the existence of certain undesirable substructures of the corresponding Tanner graph. For an AWGN channel, substructures that are called trapping sets, determine error floor performance of an LDPC code. It has been proved that absorbing sets, as a special type of trapping sets, have an important role in the error floor (see [25]).

Various combinatorial structures, including graphs, were used for a construction of LDPC codes without cycles of length four (see, e.g., [6, 16, 17, 23]). In [7], the authors investigated a family of LDPC codes constructed by taking bipartite cubic symmetric graphs as the Tanner graphs. In this paper, we construct LDPC codes from cubic semisymmetric graphs and study the smallest absorbing sets in the corresponding Tanner graphs.

The paper is organized as follows. In Section 2, the construction of the family of LDPC codes using cubic semisymmetric graphs is presented, some properties of the obtained codes are analyzed and the results regarding the code parameters are given. Furthermore, the expression for the variance of the syndrome weight of the constructed LDPC codes is presented. In Section 3, the structure of the smallest absorbing sets is studied. Sections 4 and 5 contain computational and simulation results. 


\section{LDPC codes constructed from cubic semisymmetric graphs}

Let $\mathcal{G}$ be a connected cubic semisymmetric graph with $2 n$ vertices, and denote by $A$ its adjacency matrix. Since every semisymmetric graph is bipartite with two parts of equal size, its adjacency matrix can be written as follows

$$
A=\left[\begin{array}{cc}
0 & H \\
H^{T} & 0
\end{array}\right],
$$

where $H$ is an $n \times n$ matrix.

Taking the matrix $H$ as a parity-check matrix, one can construct a $(3,3)$-regular LDPC code $\mathcal{C}_{H}(\mathcal{G})$ of length $n$. The dimension of that code is equal to $n-\operatorname{rank}_{2}(H)$, where $\operatorname{rank}_{2}(H)=\frac{1}{2} \operatorname{rank}_{2}(A)$. Furthermore, the density of the parity-check matrix $H$ is equal to $\frac{3}{n}$. For the constructed code $\mathcal{C}_{H}(\mathcal{G})$, the cubic semisymmetric graph $\mathcal{G}$ is its Tanner graph.

From the fact that semisymmetric graphs are edge-transitive, but not vertex-transitive, it follows that $H^{T}$ determines another LDPC code $\mathcal{C}_{H^{T}}(\mathcal{G})$. The code $\mathcal{C}_{H^{T}}(\mathcal{G})$ is a $(3,3)$-regular LDPC code of length $n$, and its dimension is equal to $n-\operatorname{rank}_{2}(H)$ as well.

Let $H$ and $H^{T}$ be $n \times n$ parity-check matrices of the codes $\mathcal{C}_{H}(\mathcal{G})$ and $\mathcal{C}_{H^{T}}(\mathcal{G})$, respectively. For the code $\mathcal{C}_{H}(\mathcal{G})$, the bit node graph $\Gamma_{b}$ is defined in the following way: vertices of the graph correspond to codeword bits, and two vertices are adjacent if and only if the corresponding bits are included in the same parity-check equation. In other words, two vertices of the graph $\Gamma_{b}$ are adjacent if and only if the corresponding bit nodes of the Tanner graph of the code $\mathcal{C}_{H}(\mathcal{G})$ have a common neighbour. Similarly, the vertices of the check node graph $\Gamma_{c}$ correspond to parity-check equations of the code, and two vertices are adjacent if and only if the corresponding parity-check equations have a bit in common. That is to say, two vertices of the graph $\Gamma_{c}$ are adjacent if and only if the corresponding check nodes of the Tanner graph of the $\operatorname{code} \mathcal{C}_{H}(\mathcal{G})$ have a common neighbour. Note that the check node graph $\Gamma_{c}$ of the $\operatorname{code} \mathcal{C}_{H}(\mathcal{G})$ is the bit node graph of the code $\mathcal{C}_{H^{T}}(\mathcal{G})$.

Theorem 2.1. Let $\mathcal{G}$ be a connected cubic semisymmetric graph with girth at least six and let $H$ be the parity-check matrix of the code $\mathcal{C}_{H}(\mathcal{G})$. Then the corresponding bit node graph $\Gamma_{b}$ and check node graph $\Gamma_{c}$ are 6-regular.

Proof. Let $v$ be a bit node of the Tanner graph $\mathcal{G}$. The degree of the node $v$ is equal to three, and each of its neighbours is adjacent to another two bit nodes. Using the fact that $\mathcal{G}$ does not have cycles of length four, it follows that $v$ has a common neighbour with exactly six other bit nodes. In other words, the degree of a vertex of the graph $\Gamma_{b}$ is equal to six, i.e., the graph $\Gamma_{b}$ is 6 -regular. In the same way it can be concluded that the graph $\Gamma_{c}$ is also 6-regular.

Theorem 2.2. Let $\mathcal{G}$ be a connected cubic semisymmetric graph with $2 n$ vertices and girth at least six. Further, let $H$ be the parity-check matrix of the code $\mathcal{C}_{H}(\mathcal{G})$ and let $\Gamma_{b}$ and $\Gamma_{c}$ be the corresponding bit node graph and check node graph, respectively. Matrices $T_{b}$ and $T_{c}$ are square $(0,1)$-matrices of order $n$ satisfying $T_{b}=H^{T} H-3 I$ and $T_{c}=H H^{T}-3 I$ if and only if $T_{b}$ and $T_{c}$ are the adjacency matrices of the graphs $\Gamma_{b}$ and $\Gamma_{c}$, respectively. 
Proof. Let us consider the $n \times n$ matrix $H^{T} H=\left[h_{i, j}\right]$. The degree of a bit node of the Tanner graph $\mathcal{G}$ of the code $\mathcal{C}_{H}(\mathcal{G})$ is equal to three, hence $h_{i, i}=3, i \in\{1, \ldots, n\}$. An element $h_{i, j}, i \neq j$, of the matrix $H$ is equal to one or zero depending on whether the corresponding nodes of the graph $\Gamma_{b}$ are adjacent or not. Accordingly, $T_{b}=H^{T} H-3 I$, where $T_{b}$ is the adjacency matrix of the graph $\Gamma_{b}$.

Conversely, let $T_{b}=\left[t_{i, j}\right]$ be an $n \times n(0,1)$-matrix with the property that $T_{b}=H^{T} H-3 I . H^{T} H$ is a symmetric matrix and, consequently, $T_{b}$ is also a symmetric matrix such that $t_{i, i}=0, i \in\{1, \ldots, n\}$. The girth of the Tanner graph $\mathcal{G}$ is greater than four, so $h_{i, j}, i \neq j$, is equal to zero or one, and represents the number of common neighbours of the corresponding bit nodes of the Tanner graph $\mathcal{G}$ of the code $\mathcal{C}_{H}(\mathcal{G})$. It follows that $T_{b}$ is the adjacency matrix of the graph $\Gamma_{b}$.

An analog statement for the matrix $T_{c}$ can be formed similarly by observing check nodes of the Tanner graph of the code $\mathcal{C}_{H}(\mathcal{G})$.

A clique of a graph $G$ is a complete subgraph of the graph $G$. The clique number of the graph $G$, denoted by $\omega(G)$, is the number of vertices in a clique of the largest size in $G$, i.e. the order of a complete subgraph of $G$ of maximum possible size for $G$. In the sequel, the clique number of the bit node graph $\Gamma_{b}$ and the check node graph $\Gamma_{c}$ will be examined.

Lemma 2.3. Let $\mathcal{G}$ be a connected cubic semisymmetric graph. Further, let $\mathcal{C}_{H}(\mathcal{G})$ be the corresponding LDPC code and let $\Gamma_{b}$ and $\Gamma_{c}$ be its bit node and check node graph, respectively. The clique numbers of the graphs $\Gamma_{b}$ and $\Gamma_{c}$ are at least three.

Proof. Each check node of the Tanner graph $\mathcal{G}$ is a common neighbour of every pair of its three adjacent bit nodes. Thus, each check node determines the complete graph $K_{3}$ as a subgraph of the bit node graph $\Gamma_{b}$. Similarly, each bit node of the Tanner graph determines the complete graph $K_{3}$ as a subgraph of the check node graph $\Gamma_{c}$. Hence, $\omega\left(\Gamma_{b}\right), \omega\left(\Gamma_{c}\right) \geq 3$.

Lemma 2.4. Let $\mathcal{G}$ be a connected cubic semisymmetric graph with girth greater than six. Further, let $\mathcal{C}_{H}(\mathcal{G})$ be the corresponding LDPC code and let $\Gamma_{b}$ and $\Gamma_{c}$ be its bit node and check node graph, respectively. Then the complete graph $K_{4}$ is not a subgraph of $\Gamma_{b}$ or $\Gamma_{c}$.

Proof. Suppose that $K_{4}$ is a subgraph of the graph $\Gamma_{b}$. Let the bit nodes $u_{1}, u_{2}, u_{3}, u_{4}$ be the vertices of $K_{4}$. We have the following two possibilities:

(a) One of the check nodes (say $v_{1}$ ) in the corresponding subgraph of the Tanner graph $\mathcal{G}$ has degree three. Let $u_{1}, u_{2}$ and $u_{3}$ be the bit nodes adjacent with $v_{1}$. Furthermore, let the check node $v_{2}$ be a common neighbour of $u_{1}$ and $u_{4}$. Since $u_{2}$ and $u_{4}$ are adjacent in $\Gamma_{b}$, they have a common neighbour $v_{3}$ in $\mathcal{G}$. Then $u_{1} v_{1} u_{2} v_{3} u_{4} v_{2} u_{1}$ is a cycle of length six, which is impossible since the girth of the graph $\mathcal{G}$ is greater than six.

(b) The check nodes in the corresponding subgraph of the Tanner graph $\mathcal{G}$ have degrees at most two. Let the check node $v_{i}$ be a common neighbour of the bit nodes $u_{1}$ and $u_{i+1}, i=1,2,3$. Since $u_{2}$ and $u_{4}$ are adjacent in $\Gamma_{b}$, they have a common neighbour $v_{4}$ in $\mathcal{G}$. Then $u_{1} v_{1} u_{2} v_{4} u_{4} v_{3} u_{1}$ is a cycle of length six, which contradicts the fact that the girth of the graph $\mathcal{G}$ is greater than six. 
Analog arguments yield that $K_{4}$ is not a subgraph of $\Gamma_{c}$.

The following theorem is a direct consequence of Lemmas 2.3 and 2.4.

Theorem 2.5. Let $\mathcal{G}$ be a connected cubic semisymmetric graph with girth greater than six. Further, let $\mathcal{C}_{H}(\mathcal{G})$ be the corresponding LDPC code and let $\Gamma_{b}$ and $\Gamma_{c}$ be its bit node and check node graph, respectively. Then $\omega\left(\Gamma_{b}\right)=\omega\left(\Gamma_{c}\right)=3$.

In the sequel, we discuss the minimum distance of the codes $\mathcal{C}_{H}(\mathcal{G})$ and $\mathcal{C}_{H^{T}}(\mathcal{G})$. The following results from [24] will be used.

Theorem 2.6 ([24, Theorem 3.1]). Let $\mathcal{C}$ be a binary linear code with a parity-check matrix $H$. Then there exists a codeword in $\mathcal{C}$ with weight $w$ if and only if there are $w$ columns in $H$ whose vector sum is a zero vector.

Theorem 2.7 ([24, Theorem 3.2]). Let $\mathcal{C}$ be a binary linear code with a parity-check matrix $H$. Then the minimum distance of the code $\mathcal{C}$ is equal to the smallest number of columns in $H$ whose vector sum is a zero vector.

The column weight of parity check matrices $H$ and $H^{T}$ of codes $\mathcal{C}_{H}(\mathcal{G})$ and $\mathcal{C}_{H^{T}}(\mathcal{G})$ is equal to three, and according to Theorem 2.6, the codes are even. Therefore, the minimum distance of the codes is an even number.

Theorem 2.8. Let $\mathcal{G}$ be a connected cubic semisymmetric graph with girth greater than six. Let $d\left(\mathcal{C}_{H}(\mathcal{G})\right)$ and $d\left(\mathcal{C}_{H}^{T}(\mathcal{G})\right)$ be the minimum distances of the codes $\mathcal{C}_{H}(\mathcal{G})$ and $\mathcal{C}_{H^{T}}(\mathcal{G})$, respectively. Then $d\left(\mathcal{C}_{H}(\mathcal{G})\right) \geq 6$ and $d\left(\mathcal{C}_{H}^{T}(\mathcal{G})\right) \geq 6$.

Proof. The column weight of the parity-check matrix $H$ of the code $\mathcal{C}_{H}(\mathcal{G})$ is equal to three, and since the graph $\mathcal{G}$ does not have cycles of length four, it follows that the minimum distance of the code is at least four (see [13]). Assume that the minimum distance of the code is equal to four. As a consequence of Theorem 2.7, four columns of the parity-check matrix whose sum equals zero exist. Therefore, a set $S$ in the graph $\mathcal{G}$, which consists of four bit nodes such that each pair of the vertices has a different common neighbour in $\mathcal{G}$, exists. Moreover, the set $S$ determines the complete graph $K_{4}$ as a subgraph of the bit node graph $\Gamma_{b}$. Using Theorem 2.5, we conclude that the minimum distance of the code is at least six.

Observing check nodes of the Tanner graph of the code $\mathcal{C}_{H}(\mathcal{G})$, and the check node graph $\Gamma_{c}$, one can prove the statement for the minumum distance of the code $\mathcal{C}_{H^{T}}(\mathcal{G})$.

In [7, Theorem 1], the minimum distance of an LDPC code constructed from a bipartite cubic symmetric graph is expressed using the second largest eigenvalue of the adjacency matrix of that graph. In a similar way, using the result given in Theorem 2.8, one can prove the following theorem.

Theorem 2.9. Let $\mathcal{G}$ be a connected cubic semisymmetric graph with $2 n$ vertices and girth greater than six. Let $\lambda_{2}$ be the second largest eigenvalue of its adjacency matrix $A$. Let $d\left(\mathcal{C}_{H}(\mathcal{G})\right)$ and $d\left(\mathcal{C}_{H}^{T}(\mathcal{G})\right)$ be the minimum distances of the codes $\mathcal{C}_{H}(\mathcal{G})$ and $\mathcal{C}_{H^{T}}(\mathcal{G})$, respectively. Then the following inequalities hold

$$
d \geq \begin{cases}\frac{2}{5} n, & \lambda_{2} \leq 2, \\ \frac{2}{9} n, & 2<\lambda_{2} \leq \sqrt{6}, \\ 6, & \sqrt{6}<\lambda_{2}<3\end{cases}
$$


where $d \in\left\{d\left(\mathcal{C}_{H}(\mathcal{G})\right), d\left(\mathcal{C}_{H}^{T}(\mathcal{G})\right)\right\}$.

Remark 2.10. The results given above refer to the LDPC codes constructed from connected cubic semisymmetric graphs with girth greater than six. According to the classification of cubic semisymmetric graphs with at most 768 vertices (see [4]), all such graphs have girth at least eight. Consequently, all of the associated LDPC codes have properties stated above.

Theorem 2.11. Let $\mathcal{G}$ be a connected cubic semisymmetric graph with $2 n$ vertices. Then the dimension of the codes $\mathcal{C}_{H}(\mathcal{G})$ and $\mathcal{C}_{H^{T}}(\mathcal{G})$ is at most $n-2 \alpha\left(\Gamma_{b}\right)+1$, where $\alpha\left(\Gamma_{b}\right)$ is the independence number of the bit node graph $\Gamma_{b}$.

Proof. The 2-rank of the parity-check matrix of a binary code determines its dimension. The 2-rank of the matrix $H$ is equal to the 2-rank of the matrix $H^{T}$ and, therefore, it is sufficent to observe the matrix $H$ and the corresponding code $\mathcal{C}_{H}(\mathcal{G})$. A maximal independent set of $\Gamma_{b}$ determines $\alpha\left(\Gamma_{b}\right)$ linearly independent columns of the parity check matrix $H$. These columns have the property that no two columns have an entry equal to one at the same position. Due to the fact that $\Gamma_{b}$ is a 6-regular graph, there are $6 \alpha\left(\Gamma_{b}\right)$ ones at different positions within the columns. Therefore, adding any other $\alpha\left(\Gamma_{b}\right)-1$ columns of the matrix, a set of $2 \alpha\left(\Gamma_{b}\right)-1$ linearly independent columns of the parity check matrix is defined. Hence, 2-rank of the matrix $H$ is at least $2 \alpha\left(\Gamma_{b}\right)-1$.

As a consequence, the dimension of the code is at most $n-2 \alpha\left(\Gamma_{b}\right)+1$, where $n$ is the length of the code, i.e. the number of vertices of the graph $\Gamma_{b}$, and $\alpha\left(\Gamma_{b}\right)$ is the independence number of the graph $\Gamma$.

\subsection{The variance of syndrome weight}

To predict a decoding efficiency one can use a channel state information (CSI) (e.g. the crossover probability, a signal-to-noise ratio), which has an important role for communication systems. The estimation (performed prior to decoding) of the crossover probability based on the probability of syndrome weight was proposed in [18] and [27].

The expression for the variance of the syndrome weight of the LDPC codes constructed from bipartite cubic symmetric graphs is given in [7]. In a similar way, one can obtain the expression for the variance of the syndrome weight of the LDPC codes constructed from cubic semisymmetric graphs which is given by

$$
\operatorname{Var}(w)=\frac{n}{2}\left(7 f_{6}(\rho)-6 f_{4}(\rho)\right),
$$

where the function $f_{t}$ is defined by $f_{t}(\rho)=\frac{1-(1-2 \rho)^{t}}{2}$ (see [22]).

\section{Absorbing sets}

Let $G=G(C)$ be the Tanner graph of an LDPC code $C$ which is determined by an $m \times n$ parity check matrix $H$. A $(\kappa, \tau)$ trapping set is a set $T$, that consists of $\kappa$ bit nodes, having the property that the induced subgraph $G[T]$ has exactly $\tau$ check nodes of odd degree. The most harmful trapping sets are those with small sizes and small ratios $\frac{\tau}{\kappa}$. If the Tanner graph of an LDPC code does not have trapping sets with size smaller than the minimum distance of the code, then the error floor of the code is dominated by the minimum distance (see [9]). Let $T$ be a trapping set. If every bit node in $G[T]$ is connected with fewer check nodes of odd degree than check nodes of even degree, then $T$ is called an absorbing set. 
Let $A$ be a $(\kappa, \tau)$ - trapping set in the Tanner graph of an $\left(3, w_{r}\right)$ LDPC code. Using simple counting it can be seen that $\tau$ is an even number if $\kappa$ is even, and an odd number if $\kappa$ is odd.

The results in the sequel refer to the LDPC codes for which the corresponding Tanner graphs have girth at least six. We examine the existence of the smallest absorbing sets in the Tanner graphs of the LDPC codes constructed from the cubic semisymmetric graphs.

Theorem 3.1. Let the Tanner graph of the LDPC code $\mathcal{C}_{H}(\mathcal{G})$ be a connected cubic semisymmetric graph $\mathcal{G}$ with girth at least six. Then there is no absorbing set of size smaller than three in the graph $\mathcal{G}$.

Proof. The proof follows directly from the definition of an absorbing set and the fact that the Tanner graph of the code has no cycles of length four.

Theorem 3.2. Let $\mathcal{G}$ be a connected cubic semisymmetric graph with girth greater than six, which is the Tanner graph of the $L D P C$ codes $\mathcal{C}_{H}(\mathcal{G})$ and $\mathcal{C}_{H^{T}}(\mathcal{G})$. The Tanner graph $\mathcal{G}$ has no absorbing set of size three.

Proof. Let $A$ be a $(3,3)$-absorbing set, which is the only possible structure of an absorbing set of size three in the Tanner graph of the codes (see Figure 1). The proof follows directly from the fact that the absorbing set defines a cycle of length six in the Tanner graph.

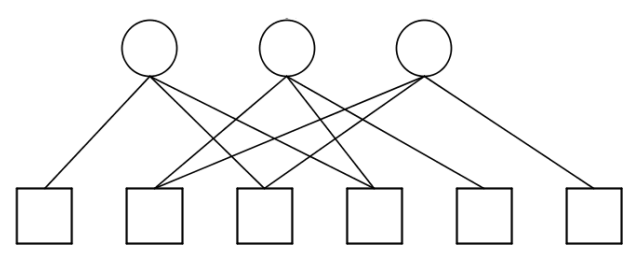

Figure 1: The only possible structure of an absorbing set of size three in the Tanner graph of the LDPC codes $\mathcal{C}_{H}(\mathcal{G})$. and $\mathcal{C}_{H^{T}}(\mathcal{G})$.

Theorem 3.3. Let $\mathcal{G}$ be a connected cubic semisymmetric graph with girth greater than six, which is the Tanner graph of the LDPC codes $\mathcal{C}_{H}(\mathcal{G})$ and $\mathcal{C}_{H^{T}}(\mathcal{G})$. The only possible structure for an absorbing set of size four is $(4,4)$-absorbing set.

Proof. Since the size of an absorbing set is an even number, and according to the previous observations, the possible structures for absorbing sets of size four in the Tanner graph of the codes are $(4,0),(4,2)$ and $(4,4)$ absorbing sets (see Figure 2(a), (b) and (c), respectively). The proof follows directly from the fact that $(4,0)$ and $(4,2)$ absorbing sets define the complete graph $K_{4}$ as a subgraph of the graph $\Gamma_{b}$. 
(a)

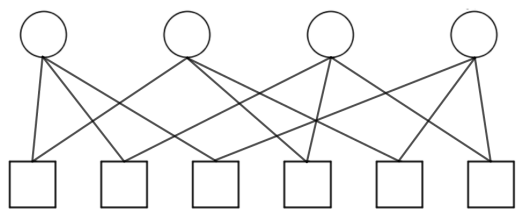

(b)

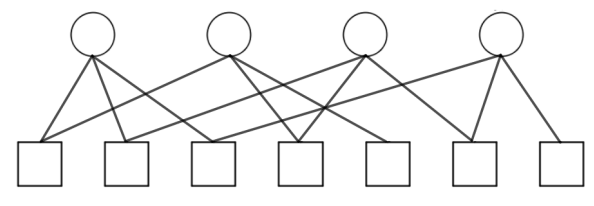

(c)

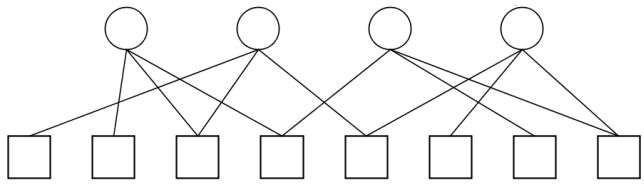

Figure 2: The possible structures of an absorbing set of size four in the Tanner graph of the LDPC codes $\mathcal{C}_{H}(\mathcal{G})$ and $\mathcal{C}_{H^{T}}(\mathcal{G})$.

\section{Computational results}

Within this section the parameters of the LDPC codes obtained from cubic semisymmetric graphs are presented. For the construction of the cubic semisymmetric graphs we have employed the method presented in [1]. The parameters of the constructed codes can be seen in Table 1. The parameter $v$ denotes the number of vertices of the corresponding cubic semisymmetric graph.

\begin{tabular}{|c|c|c|c|c|c|}
\hline$v$ & $\mathbf{L D P C}_{1}$ & $\mathbf{L D P C}_{2}$ & $v$ & $\mathrm{LDPC}_{1}$ & $\mathbf{L D P C}_{2}$ \\
\hline 54 & {$[27,8,6]$} & {$[27,8,8]^{*}$} & 448 & {$[224,33,32]$} & {$[224,33,32]$} \\
\hline 112 & {$[56,12,14]$} & {$[56,12,16]$} & 486 & {$[243,2,162]^{*}$} & {$[243,2,162]^{*}$} \\
\hline 120 & {$[60,14,8]$} & {$[60,14,12]$} & 546 & {$[273,5,130]$} & {$[273,5,130]$} \\
\hline 144 & {$[72,16,12]^{*}$} & {$[72,16,14]^{*}$} & 576 & {$[288,32,48]$} & {$[288,32,56]$} \\
\hline 216 & {$[108,16,24]$} & {$[108,16,32]$} & 672 & {$[336,47,14]$} & {$[336,47,42]$} \\
\hline 240 & {$[120,22,16]$} & {$[120,22,24]$} & 702 & {$[351,8,78]$} & {$[351,8,104]^{*}$} \\
\hline 294 & {$[147,26,14]$} & {$[147,26,26]$} & 720 & {$[360,10,120]$} & {$[360,10,120]$} \\
\hline 336 & {$[168,24,14]$} & {$[168,24,42]$} & 784 & {$[392,12,98]$} & {$[392,12,112]$} \\
\hline 378 & {$[189,11,42]$} & {$[189,11,56]$} & 798 & {$[399,5,190]$} & {$[399,5,190]$} \\
\hline 384 & {$[192,35,16]$} & {$[192,35,18]$} & 864 & {$[432,32,96]$} & {$[432,32,108]$} \\
\hline 400 & {$[200,24,32]$} & {$[200,24,60]$} & 882 & {$[441,44,42]$} & {$[441,44,78]$} \\
\hline 432 & {$[216,24,48]$} & {$[216,24,60]$} & 896 & {$[448,48,84]$} & {$[448,48,100]$} \\
\hline
\end{tabular}

Table 1: The parameters of LDPC codes constructed from cubic semisymmetric graphs with less than 1000 vertices (using the method presented in [1]). 
The Tanner graphs of the constructed codes have girth at least eight. The codes $\mathcal{C}_{H}(\mathcal{G})$ and $\mathcal{C}_{H^{T}}(\mathcal{G})$ are isomorphic in the case when the number of vertices of the cubic semisymmetric graph $G$ is $486,546,720$ or 798 .

Remark 4.1. Lately, much interest has been devoted to LCD codes, which have an important application in cryptography, in protection against side-channel and fault attacks (see [2]). Self-orthogonal codes can be used to construct quantum error-correcting codes, which can protect quantum information in quantum computations and quantum communications (see [3]).

The LDPC codes marked in bold are self-orthogonal codes, and those labeled with * in Table 1 are LCD codes.

Remark 4.2. Codes $\mathcal{C}_{H}(\mathcal{G})$ and $\mathcal{C}_{H^{T}}(\mathcal{G})$ constructed from a cubic semisymmetric graph (CSSG) have the same length and dimension, and, in general, different minimum distance. Thus, the construction gives diversity in code parameters for the same graph, which is not the case for LDPC codes which are constructed in [7] using cubic symmetric graphs (CSGs).

According to the classification of CSSGs with at most 768 vertices (see [4]), all the graphs have girth at least eight, while according to [5] many CSGs have girth equal to six. Moreover, semisymmetric graphs form a wider family than symmetric graphs.

Furthermore, we have compared the parameters of the LDPC codes constructed from CSSGs to the parameters of the LDPC codes constructed from CSGs. The results are shown in Table 2. It can be concluded that, for the same code length, the LDPC codes from CSSGs achieve higher code rate than those constructed using CSGs. When $n=27$, the code rate is four times greater.

\begin{tabular}{|c|c|c|}
\hline$n$ & Rate (CSSG) & Rate (CSG) \\
\hline 27 & 0.296 & 0.074 \\
56 & 0.214 & $\{0.107,0.143\}$ \\
60 & 0.233 & $\{0.067,0.083\}$ \\
72 & 0.222 & $\{0.083,0.111\}$ \\
\hline
\end{tabular}

Table 2: Rates od LDPC codes constructed from cubic symmetric and semisymmetric graphs with the same length.

\section{Simulation results}

In this section, we present simulation results of the LDPC codes derived from the cubic semisymmetric graphs, over the additive white gaussian noise (AWGN) channel. We have compared the codes with randomly generated LDPC codes of the same length and dimension and a parity-check matrix with a column weight equal to three. For randomly generated codes we have used the software for LDPC codes available on [21], which employs the construction from $[8,19]$. The codes are decoded with the sum-product decoding algorithm and the maximum number of iteration is set to 50. Figures $3-6$ show the performance of the codes. 
Remark 5.1. The LDPC codes that we are aware of were not adequate for the comparison with the LDPC codes obtained in this paper because of the different parameters of the codes. Thus, we have used the best known random construction for LDPC codes. It has been proved in [8] that the construction leads to LDPC codes with performance close to the Shannon limit. Moreover, the best results were obtained in the case of the smallest possible column weight.

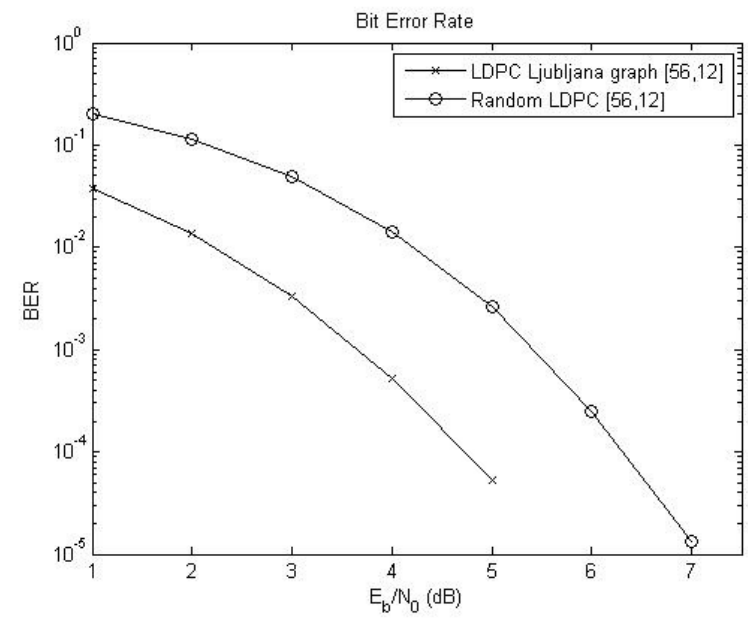

Figure 3: BER performance of the $[56,12,16]$ LDPC code derived from the Ljubljana graph.

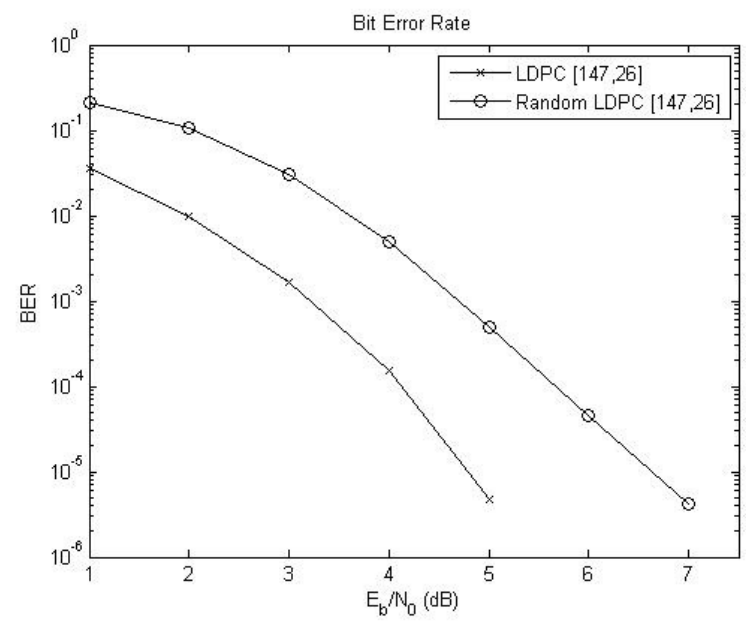

Figure 4: BER performance of the $[147,26,26]$ LDPC code derived from the cubic semisymmetric graph with 294 vertices. 


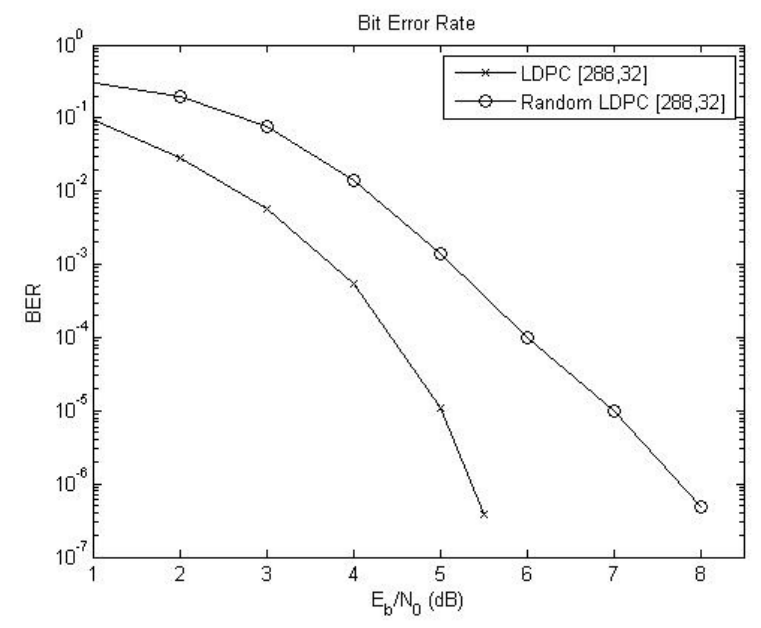

Figure 5: BER performance of the $[288,32,56]$ LDPC code derived from the cubic semisymmetric graph with 576 vertices.

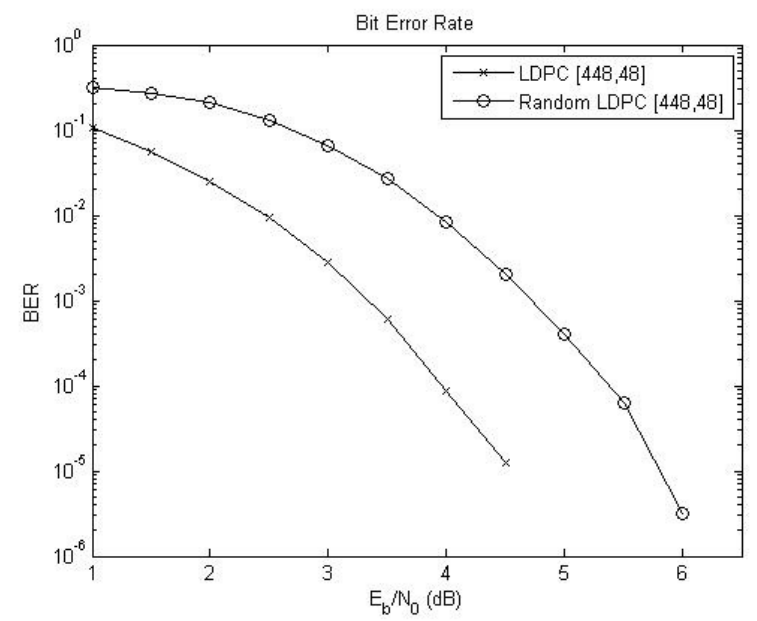

Figure 6: BER performance of the $[448,48,100]$ LDPC code derived from the cubic semisymmetric graph with 896 vertices.

The obtained simulation results indicate better BER performance of the codes constructed from the cubic semisymmetric graphs than randomly generated LDPC codes.

\section{ORCID iDs}

Dean Crnković (D) https://orcid.org/0000-0002-3299-7859

Sanja Rukavina (D) https://orcid.org/0000-0003-3365-7925

Marina Šimac (D) https://orcid.org/0000-0001-9291-3365 


\section{References}

[1] A. Bretto and L. Gillibert, G-graphs: An efficient tool for constructing symmetric and semisymmetric graphs, Discrete Appl. Math. 156 (2008), 2719-2739, doi:10.1016/j.dam.2007.11.011.

[2] J. Bringer, C. Carlet, H. Chabanne, S. Guilley and H. Maghrebi, Orthogonal direct sum masking, in: Information Security Theory and Practice. Securing the Internet of Things. WISTP 2014. Lecture Notes in Computer Science, Springer, Berlin, Heidelberg, volume 8501, 2014 pp. 40-56, doi:10.1007/978-3-662-43826-8_4.

[3] A. R. Calderbank, E. M. Rains, P. W. Shor and N. J. A. Sloane, Quantum error correction and orthogonal geometry, Phys. Rev. Lett. 78 (1997), 405-408, doi:10.1103/physrevlett.78.405.

[4] M. Conder, A. Malnič, D. Marušič and P. Potočnik, A census of semisymmetric cubic graphs on up to 768 vertices, J. Algebr. Comb. 23 (2006), 255-294, doi:10.1007/s10801-006-7397-3.

[5] M. Conder and R. Nedela, A refined classification of symmetric cubic graphs, J. Algebra 322 (2009), 722-740, doi:10.1016/j.jalgebra.2009.03.011.

[6] D. Crnković, S. Rukavina and M. Šimac, LDPC codes from $\mu$-geodetic graphs obtained from block designs, Graphs Comb. 35 (2019), 451-469, doi:10.1007/s00373-019-02007-4.

[7] D. Crnković, S. Rukavina and M. Šimac, LDPC codes constructed from cubic symmetric graphs, Appl. Algebra Eng. Commun. Comput. (2020), doi:10.1007/s00200-020-00468-2.

[8] R. M. N. D. J. C. MacKay, Near Shannon limit performance of low density parity check codes, Electron. Lett. 32 (1996), 1645-1646, doi:10.1049/el:19961141.

[9] Q. Diao, Y. Y. Tai, S. Lin and K. Abdel-Ghaffar, Trapping set structure of finite geometry ldpc codes, in: 2012 IEEE International Symposium on Information Theory Proceedings, 2012 pp. 3088-3092, doi:10.1109/isit.2012.6284130.

[10] R. Diestel, Graph Theory, Springer-Verlag, Berlin Heidelberg, 2017, doi:10.1007/ 978-3-662-53622-3.

[11] Y.-Q. Feng, M. Ghasemi and C. Wang, Cubic semisymmetric graphs of order $6 p^{3}$, Discrete Math. 310 (2010), 2345-2355, doi:10.1016/j.disc.2010.05.018.

[12] J. Folkman, Regular line-symmetric graphs, J. Comb. Theory 3 (1967), 215-232, doi:10.1016/ S0021-9800(67)80069-3.

[13] M. Greferath, C. Rößing and L. Storme, Galois geometries and low-density parity-check codes, in: Current Research Topics in Galois Geometry, Nova Science Publishers/Novinka, New York, pp. 245-270, 2014.

[14] F. Harary, Graph Theory, Addison-Wesley Publishing Company, 1969, doi:10.1201/ 9780429493768.

[15] W. C. Huffman and V. Pless, Fundamentals of Error-Correcting Codes, Cambridge University Press, Cambridge, 2003, doi:10.1017/cbo9780511807077.

[16] F. Ivanov and V. Zyablov, LDPC codes based on steiner quadruple systems and permutation matrices, in: Fourteenth International Workshop on Algebraic and Combinatorial Coding Theory, Nova Science Publishers/Novinka, New York, pp. 175-180, 2014, http: //www . moi . math.bas.bg/acct2014/acct2014end.html.

[17] J.-L. Kim, U. N. Peled, I. Perepelitsa, V. Pless and S. Friedland, Explicit construction of families of LDPC codes with no 4-cycles., IEEE Trans. Inf. Theory 50 (2004), 2378-2388, doi: 10.1109/tit.2004.834760.

[18] G. Lechner and C. Pacher, Estimating channel parameters from the syndrome of a linear code, IEEE Commun. Lett. 17 (2013), 2148-2151, doi:10.1109/lcomm.2013.091113.131646. 
[19] D. J. C. MacKay, Good error-correcting codes based on very sparse matrices, IEEE Trans. Inf. Theory 45 (1999), 399-431, doi:10.1109/18.748992.

[20] A. Malnič, D. Marušič and C. Wang, Cubic edge-transitive graphs of order 2p3, Discrete Mathematics 274 (2004), 187-198, doi:10.1016/S0012-365X(03)00088-8.

[21] R. M. Neal, Software for Low Density Parity Check (LDPC) codes, University of Toronto, 2012, https: / /www. cs.toronto. edu/ radford/ldpc. software.html.

[22] C. Pacher, P. Grabenweger and D. E. Simos, Weight distribution of the syndrome of linear codes and connections to combinatorial designs, in: 2016 IEEE International Symposium on Information Theory (ISIT), IEEE Press, 2016 pp. 3038-3042, doi:10.1109/isit.2016.7541857.

[23] J. Rosenthal and P. O. Vontobel, Constructions of LDPC codes using Ramanujan graphs and ideas from Margulis, in: 38th Allerton Conference on Communication, Control and Computing, 2000 pp. 248-257, https://www.math.uzh.ch/aa/index.php? publication-show\&key1=Coding 20 Theory\&key2=Joachim:20Rosenthal.

[24] W. E. Ryan and S. Lin, Channel codes. Classical and modern, Cambridge University Press, 2009, doi:10.1017/cbo9780511803253.

[25] C. Schlegel and S. Zhang, On the dynamics of the error floor behavior in (regular) LDPC codes, IEEE Trans. Inf. Theory 56 (2010), 3248-3264, doi:10.1109/tit.2010.2048448.

[26] R. M. Tanner, A recursive approach to low complexity codes, IEEE Trans. Inf. Theory 27 (1981), 533-547, doi:10.1109/tit.1981.1056404.

[27] V. Toto-Zarasoa, A. Roumy and C. Guillemot, Maximum likelihood BSC parameter estimation for the Slepian-Wolf problem, IEEE Commun. Lett. 15 (2011), 232-234, doi:10.1109/lcomm. 2011.122810.102182. 